\title{
Kaposi's Sarcoma-Associated Herpesvirus Viral Interferon Regulatory Factor Confers Resistance to the Antiproliferative Effect of Interferon-a
}

\author{
C. Clay Flowers, Scarlett P. Flowers, and Gary J. Nabel \\ Howard Hughes Medical Institute, University of Michigan Medical \\ Center, Departments of Internal Medicine and Biological Chemistry, \\ Ann Arbor, Michigan, U.S.A. \\ Communicated by J. Leiden. Accepted April 15, 1998.
}

\begin{abstract}
Background: Kaposi's sarcoma-associated herpesvirus (KSHV) encodes a 442 amino acid polypeptide-designated viral interferon regulatory factor (vIRF) that displays homology to members of the interferon regulatory factor (IRF) family that bind to consensus interferon sequences and transactivate cellular genes that can modulate growth inhibition. Studies were conducted to determine whether vIRF affects the growth suppression mediated by interferon- $\alpha$ (IFN- $\alpha$ ) in a human B lymphocyte cell line.

Materials and Methods: The human B lymphocyte cell line Daudi, which is sensitive to the antiproliferative effects of IFN- $\alpha$, was stably transfected to express vIRF, and the proliferative response of vIRF expressing cells to IFN- $\alpha$ was compared with controls. The effect of vIRF on IRF-1 transactivation was analyzed by co-transfection of an IFN- $\alpha$-responsive chloramphenicol acetyltransferase reporter and expression plasmids encoding IRF-1 and vIRF. Electrophoretic mobility shift assays were con-
\end{abstract}

ducted to determine whether vIRF interferes with the DNA binding activity of IRF-1.

Results: Daudi human B lymphocyte cells expressing vIRF were resistant to the antiproliferative effects of IFN- $\alpha$, whereas wild-type Daudi or Daudi cells transformed with vector DNA were growth inhibited by IFN- $\alpha$. The activation of an interferon-responsive reporter by IFN- $\alpha$ or IRF-1 was repressed by expression of vIRF. IRF-1 DNA binding activity was unaffected by VIRF, and VIRF alone did not bind to the interferon consensus sequence.

Conclusions: These studies revealed that vIRF functions to inhibit interferon-mediated growth control of a human B lymphocyte cell line by targeting IRF-1 transactivation of interferon-inducible genes. Since KSHV is a B lymphotropic herpesvirus associated with two forms of $B$ lymphocyte neoplasms, these effects of vIRF likely contribute to B cell oncogenesis associated with KSHV infection.

\section{Introduction}

Kaposi's sarcoma (KS)-associated herpesvirus (KSHV) or human herpesvirus-8 is associated with all epidemiological forms (AIDS-related, classical, post-transplant, and endemic) of Kaposi's sarcoma (1-6). Epidemiological studies dem-

Address correspondence and reprint requests to: Dr. Gary J. Nabel, Howard Hughes Medical Institute, University of Michigan Medical Center, Departments of Internal Medicine and Biological Chemistry, $1150 \mathrm{~W}$. Medical Center Drive, 4520 MSRB I, Ann Arbor, MI 48109-0650, U.S.A. Phone: 734-647-4798; Fax: 734-647-4730; E-mail: gnabel@umich.edu onstrate that seroconversion to KSHV antibodies against virally encoded latent nuclear antigens occurs prior to the onset of KS (7-9) and that KSHV seropositivity is not common among the general population but is present in groups at risk for the development of KS (10-12). In KS biopsies, KSHV is present in endothelial cells and spindle cells (13-15), and viral gene expression is restricted; however, some cells are productively infected, as evidenced by the presence of viral particles (16) and by transmission of virus from KS biopsy material to cell lines susceptible to 
infection (17). In addition, KSHV infects $\mathrm{CD} 19^{+}$ B lymphocytes $(18,19)$ and has been detected in cells of this lineage that were derived from body cavity-based lymphomas (20-22) and multicentric Castleman's disease (23).

Sequencing of the KSHV genome (24) revealed similarity to gammaherpesviruses. These include herpesvirus saimiri, which causes fulminate $\mathrm{T}$ lymphomas in primates other than its natural host, the squirrel monkey (reviewed in ref. 25), and Epstein-Barr virus (EBV), which is associated with nasopharyngeal carcinoma and Burkitt's lymphoma (reviewed in ref. 26). The herpesvirus saimiri transformation-associated protein of strain C (Stp-C) $(27,28)$ and the EBV nuclear antigens (EBNAs) and latent membrane proteins (LMPs) contribute to immortalization and/or transformation by these herpesviruses. Interestingly, KSHV does not encode proteins with homology to either herpesvirus saimiri or EBV transformation-associated proteins; however, KSHV does encode proteins that may affect cellular signaling pathways and the regulation of cell growth (24). For example, expression of the KSHV homologue of human cyclin D in cultured cells induces phosphorylation and inactivation of the retinoblastoma tumor suppressor protein $(29,30)$. The KSHV homologue of bcl-2 inhibits bax-mediated apoptosis (31), and KSHV ORF $\mathrm{K} 13$, based on its homology with other viral proteins with anti-apoptotic properties, may function to inhibit Fas and tumor necrosis factor-1 (TNF-1) receptor signaling pathways that induce programmed cell death $(32,33)$. In addition, the KSHV homologue of interleukin-6 (IL-6) (vIL-6) supports the growth of IL-6-dependent cell lines that undergo apoptosis in the absence of IL-6 (18). vIL-6 gene expression has been detected in KSHV-infected dendritic cells derived from multiple myeloma patients, suggesting that KSHV may play a role in multiple myeloma via paracrine stimulation of bone marrow plasma cells by vIL-6 (34).

Another KSHV polypeptide that may affect cellular signaling pathways is viral IFN regulatory factor (VIRF) encoded by ORF K9; this viral protein displays homology to members of the IFN regulatory factor family (IRFs; 35,36 ). In response to cytokines such as class I or class II IFNs or virus infection, IRFs regulate transcription of a broad range of genes involved in immune regulation, cell growth control, and the establishment of an antiviral state (reviewed in ref. 36). IFN regulatory factor-1 (IRF-1) binds to the IFN consensus sequence [ICS; (G/A)(G/C)
TTTC] and activates transcription of IFN-inducible genes such as IFN- $\alpha$ and IFN- $\beta$ (37). IRF-2 and IRF-1 possess homologous amino-terminal DNA binding domains, and IRF-2 binds to the IFN- $\alpha$ ICS with affinity equivalent to that of IRF-1 (38)-IRF-2 and functions to repress expression of IFN-inducible genes by competing with IRF-1 for ICS binding sites with equal affinity $(35,38,39)$. Two other members of the IRF family, IFN consensus sequence-binding protein (ICSBP) and IFN consensus sequence-binding protein of activated Tymphocytes (ICSAT) function to repress gene expression induced by IRF-1 or IFNs. Thus, IFN-stimulated gene expression mediated by IRF-l is controlled negatively by multiple IRFs. Recent studies have revealed that KSHV VIRF inhibits IFN signaling in a manner similar to that of the cellular repressor IRFs $(40,41)$. These studies demonstrated that transient expression of vIRF inhibited IFN- or IRF-1 mediated activation of an IFN-inducible promoter. Importantly, NIH3T3 cells stably expressing vIRF were shown to display characteristics of transformed cells, and injection of these cells into nude mice induced tumor formation (41). Thus, vIRF is a transforming oncogene that may play a role in KSHV-associated neoplasms. Analyses of viral gene expression have revealed that vIRF is not expressed in Kaposi's sarcoma lesions but it is expressed in cell lines derived from body cavity-based lymphomas $(18,41)$. The studies described in this report address whether vIRF functions to inhibit IFN signaling pathways in a cell type that is relevant to KSHV cell tropism. These studies revealed that KSHV vIRF confers resistance to the antiproliferative effects of IFN- $\alpha$ in B lymphocytes.

\section{Materials and Methods}

\section{Plasmids}

A KSHV vIRF expression construct was generated by polymerase chain reaction (PCR) amplification of a DNA fragment encoding the K9 open reading frame using as template total cellular DNA isolated from the KSHV-infected cell line BCBL-1 (22). The sense primer (5'-TTTTTT GGATCCACCATGGACCCAGGCCAAAGACCGA CCCCTTTT- $3^{\prime}$ ) contained a BamHI restriction site and a Kozak sequence for translation initiation (42), while the anti-sense primer $\left(5^{\prime}\right.$-TTTTTTAA GCTTTTGCATGGCATCCCATAACGGCTTCGG$\left.3^{\prime}\right)$ contained a HindIII restriction site. Following restriction endonuclease digestion, the PCR 
product was cloned into the expression vector pMYCHIS (Invitrogen, San Diego, CA) to generate plasmid pK9MH; vIRF encoded by pK9MH contained a myc-epitope and a polyhistidine tag at the carboxyl terminus. DNA sequence analyses of both strands of pK9MH confirmed that the PCR-generated DNA fragment matched the published sequence of the $\mathrm{K} 9$ open reading frame (24). A cDNA encoding human IRF-1 was amplified by PCR using as template human spleen cDNA (GIBCO BRL, Gaithersburg, MD). The IRF-1 cDNA was cloned into a modified pcDNA3 (Invitrogen) which contains the hemagglutinin (HA) epitope, and the resulting clone, pIRF-1HA, encodes IRF-1 with an HA epitope at the amino terminus.

\section{Expression of Recombinant Proteins}

pK9MH or pIRF-1HA DNAs were transcribed and translated in vitro using TNT T7 Quick reaction rabbit reticulocyte lysates (Promega, Madison, WI) either in the presence or absence of ${ }^{35} \mathrm{~S}$-methionine according to the manufacturer's instructions. Labeled polypeptides were analyzed by fractionation on $12 \%$ SDS-PAGE. Immunoprecipitations were conducted using $0.5 \mu \mathrm{g}$ of murine monoclonal anti-myc antibody 9E10 or isotype control antibody (IgGl; Sigma, St. Louis, MO) with $20 \mu \mathrm{l}$ of in vitro translation reaction in $200 \mu \mathrm{l}$ of association buffer $(50 \mathrm{mM}$ Tris- $\mathrm{Cl}, \mathrm{pH}$ 7.4, $250 \mathrm{mM} \mathrm{NaCl}, 1 \%$ Nonidet P-40, $1 \mathrm{mM}$ dithiothreotol, and $1 \times$ concentration of protease inhibitor cocktail, Complete; Boehringer Mannheim Biochemicals, Indianapolis, IN). Antigenantibody complexes were adsorbed to protein G-agarose beads (GIBCO, BRL), washed three times with association buffer, and analyzed by SDS-PAGE. Immunoblot analyses were conducted to detect recombinant proteins in total cell extracts derived from $0.5 \times 10^{6}$ cell equivalents. Following transfer of proteins from SDSPAGE to Genescreen membranes, the filters were incubated with anti-myc antibody diluted 1:800 followed by secondary antibody conjugated to horseradish peroxidase and diluted 1:5000. Proteins were visualized using the enhanced chemiluminescence assay (Amersham, Arlington Heights, IL). Blocking and washing of filters were conducted according to the manufacturer's recommendations.

\section{Cells and Transfections}

Human embryonic 293, HeLa, and NIH3T3 cells were maintained in Dulbecco's minimal essential medium (DMEM) supplemented with penicillin $(100 \mathrm{U} / \mathrm{ml})$, streptomycin $(100 \mathrm{mg} / \mathrm{ml})$, and $5 \%$ fetal bovine serum (FBS). The calcium phosphate precipitation method (43) was used to transfect 293 cells, and cell lysates were harvested $48 \mathrm{hr}$ post-transfection. HeLa and NIH3T3 cells were transfected using Fugene- 6 transfection reagent as recommended by the supplier (Boehringer Mannheim Biochemicals). In CAT reporter assays, cell monolayers were harvested $24 \mathrm{hr}$ after infection and CAT activity was quantitated. For reporter assays of cells stimulated with IFN, cells were transfected for $12 \mathrm{hr}$ then incubated for an additional $12 \mathrm{hr}$ with recombinant IFN- $\alpha 2 \mathrm{~b}$ (Schering Corporation, Kenilworth, NJ). To correct for transfection efficiency, $3 \mu \mathrm{g}$ of RSV- $\beta$ GAL was co-transfected in each sample, and the amount of extract used in CAT assays was normalized according to the level of $\beta$-galactosidase activity (43).

\section{Cell Lines Expressing vIRF and Anti-Proliferation Assays}

Ten micrograms of linear pK9MH or pMYCHIS DNAs and $2 \times 10^{6}$ Daudi B lymphocyte cells (ATCC; ccl-213) in 0.4-cm cuvettes were pulsed using a Bio-Rad Gene pulser with settings of 220 $\mathrm{V}$ and $960 \mu \mathrm{F}$. Twenty-four hours after electroporation, geneticin (G418; Sigma) was added to the culture medium at a final concentration of $1.5 \mathrm{mg} / \mathrm{ml}$ (active concentration). Cultures were maintained in G418 selection unless employed for experimentation. For the antiproliferation assay, wild-type Daudi cells or cells transformed with pK9MH or vector DNAs were plated at a density of $4 \times 10^{4}$ cells per well in 96-well microtiter plates. The experiment was initiated by the addition of recombinant IFN- $\alpha 2 b$, and cultures received additional medium supplemented with IFN- $\alpha 2$ b on day 3 . Cell number was determined microscopically on days 3 and 5 .

\section{Electrophoretic Mobility Shift Assay}

DNA binding activity of vIRF or IRF-1 was analyzed by electrophoretic mobility gel shift assay (EMSA) using as probe a double-stranded oligonucleotide containing the PRDI site of the IFN- $\alpha$ promoter (sense-GGGAGAAGTGAAAGTG; antisense-CACTTTCACTTCTCCC) radiolabeled with $\left(\gamma-{ }^{32} \mathrm{P}\right)$ ATP. Four microliters of in vitro transcription and translation reaction charged with either pK9MH or pIRF-1HA and $1 \mathrm{~nm}$ of probe was incubated in binding buffer $(10 \mathrm{mM}$ Tris- $\mathrm{Cl}, \mathrm{pH}$ 


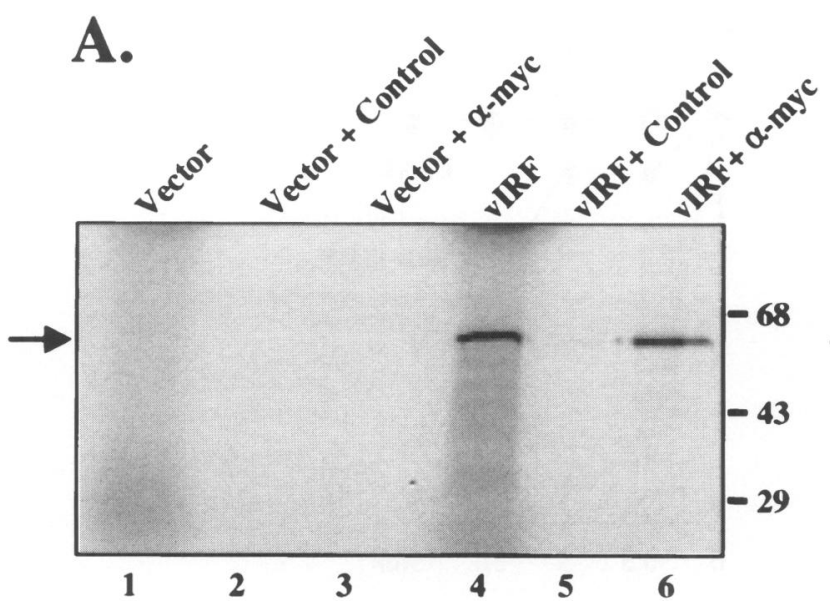

Fig. 1. Expression of vIRF in vitro and in cells transiently or stably transfected. (A) Rabbit reticulocyte lysate-coupled in vitro transcription and translation reactions (Promega) were programmed with either pMYCHIS vector DNA (lanes 1 to 3 ) or with pK9MH DNA encoding vIRF (lanes 4 to 6), and reaction products were labeled by incorporation of ${ }^{35} \mathrm{~S}$-methionine. Aliquots of the reaction were immunoprecipitated with isotype control antibody (Sigma; lanes 2 and 5) or with anti-myc antibody. In vitro translation products were fractionated by SDSPAGE and visualized by autoradiography. (B) Human embryonic 293 cells were transiently transfected by calcium phosphate precipitation method

7.5, $50 \mathrm{mM} \mathrm{NaCl}, 1 \mathrm{mM}$ EDTA, $1 \mathrm{mM}$ DTT, 4\% glycerol, $0.5 \%$ Nonidet $\mathrm{P}-40$, and $1 \mu \mathrm{m}$ of polydeoxycytosine/inosine) for $30 \mathrm{~min}$ at room temperature (44). The specificity of binding was analyzed using a 10-fold excess of competitor oligonucleotides. Protein-DNA complexes were analyzed by electrophoresis through $4 \%$ nondenaturing polyacrylamide in $0.5 \times$ Tris-borateEDTA buffer (45).

\section{Results}

Expression of vIRF In Vitro and In Vivo

To examine expression of vIRF, a plasmid encoding this gene product was employed as template in a coupled in vitro transcription and translation reaction using rabbit reticulocyte lysates (Promega) in the presence of ${ }^{35} \mathrm{~S}$-methionine. SDSPAGE analysis of the translation product revealed a band migrating at $53 \mathrm{kD}$ (Fig. 1A, lane 4 ), which corresponds in size to the predicted molecular weight of the epitope-tagged K9 ORF. A 53-kD polypeptide was not observed in reactions using vector DNA as template (Fig. 1A, lane

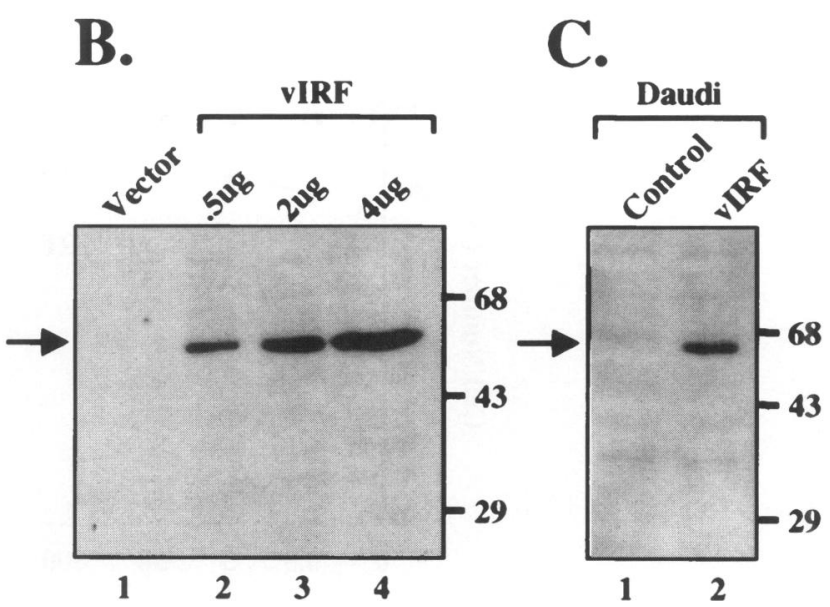

using either $4 \mu \mathrm{g}$ of pMYCHIS vector DNA (lane 1) or the indicated increasing amounts of pK9MH vIRF DNA (lanes 2 to 4). Expression of the VIRF was analyzed by immunoblot analysis of whole-cell lysates using the anti-myc antibody as probe. (C) Daudi B lymphocytes were stably transformed with either vector DNA (Control) or pK9MH DNA (vIRF) and were maintained under G418 selection for 5 weeks. Cell lysates were prepared from the pooled population of cells and were analyzed by immunoblotting using the anti-myc antibody as probe. Molecular size standards are indicated. Arrows denote the position of the $53 \mathrm{kD}$ vIRF polypeptide.

1). The presence of the myc-epitope in vIRF was confirmed by immunoprecipitation with antimyc antibody (Boehringer Mannheim Biochemicals) from the pK9MH-charged reaction (Fig. 1A, lane 6) but not from the vector DNA template reaction (Fig. 1A, lane 3 ). Isotype-matched control antibody did not immunoprecipitate the 53-kD vIRF from reactions using either vector or pK9MH DNAs as template (Fig. IA, lanes 2 and 5 , respectively). Expression of vIRF was analyzed by transient transfection of the pK9MH vIRF expression vector into the human embryonic kidney cell line, 293. Subconfluent monolayers of 293 cells were transfected with either a negative control vector alone or with different amounts of vIRF expression vector. Thirty-six hours after transfection, monolayers were harvested and cell lysates were analyzed by immunoblot analysis using anti-myc antibody followed by anti-mouse antibody conjugated to horseradish peroxidase. The 53-kD vIRF was detected in lysates from pK9MH-transfected cells (Fig. 1B, lane 2) but not from cells transfected with vector DNA (Fig. 1B, lane 1). The levels of vIRF observed in pK9MHtransfected cells corresponded to the amount of 

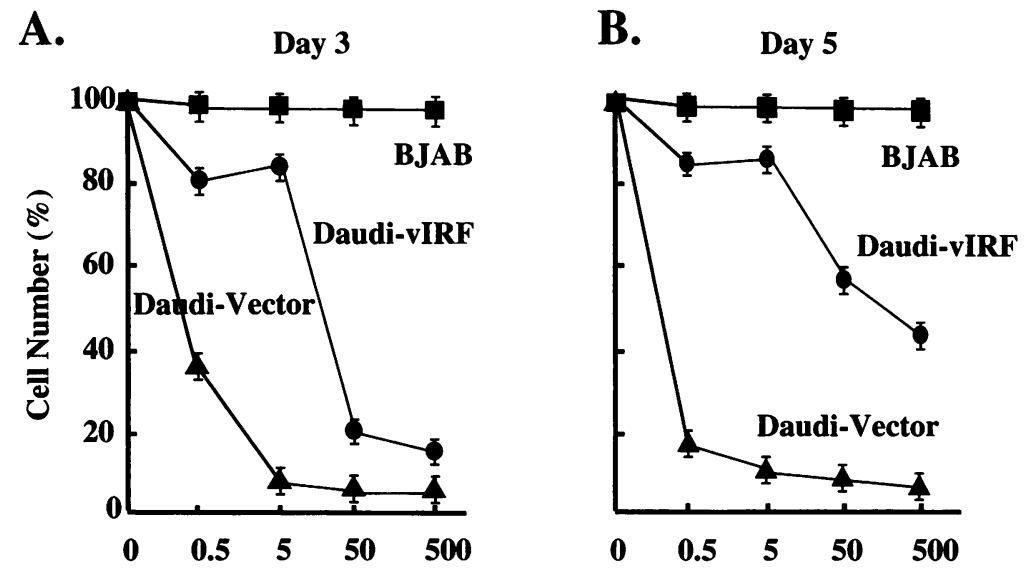

units/ml IFN- $\alpha$ 2b

Fig. 2. Daudi B lymphocytes expressing vIRF are resistant to growth inhibition mediated by IFN- $\boldsymbol{\alpha}$. Daudi B lymphocytes stably transfected with either vector DNA (Daudi-vector; triangles) or pK9MH DNA (Daudi-vIRF; circles) were treated with either normal saline or with recombinant IFN- $\alpha 2 b$ on (A) day 3 or (B) day 5. BJAB B lymphocytes (squares) were employed as a negative control for

plasmid used in each transfection (Fig. 1B, lanes 2-4). Daudi B lymphocyte cells stably transformed with pK9MH (vIRF) were analyzed by immunoblotting of cell lysates for the expression of vIRF. Using anti-myc antibody, the $53-\mathrm{kD}$ vIRF was detected in lysates from Daudi/vIRF transformed cells (Fig. 1C, lane 2). No signal corresponding to the $53-\mathrm{kD}$ protein was observed in cell lysates from cells transformed with plasmid vector (Control; Fig. 1C, lane 1).

\section{vIRF Suppresses the Anti-proliferative Response of Cells to IFN}

Because of the homology of vIRF with members of the IRF family $(18,19)$, the capacity of VIRF to alter the sensitivity of cells to IFN- $\alpha$ was analyzed. The Burkitt's lymphoma cell line Daudi is latently infected with EBV and is sensitive to growth inhibition by IFN- $\alpha(46,47)$. For the antiproliferation assay, Daudi/vIRF or Daudi/vector cells were incubated with increasing concentrations of recombinant IFN- $\alpha 2 b$ (Schering Corporation, Kenilworth, NJ). Three or five days after the addition of IFN- $\alpha 2 b$, cells in each culture were enumerated; the data are presented as a percent of the cell number of the IFN-free controls (Fig. 2). On day 3, the lowest concentration of IFN- $\alpha 2 \mathrm{~b}(0.5 \mathrm{U} / \mathrm{ml})$ substantially reduced the the effects of IFN since these cells do not respond to the antiproliferative effects of IFN- $\alpha$. Three or five days following IFN treatment, the number of viable cells in each sample was determined by microscopic analysis of cells in the presence of trypan blue. The data are expressed as a percent of the number of cells in the IFN-free controls. Each data point represents the average of three experiments.

proliferation of control Daudi cells (triangles) relative to proliferation in the absence of IFN (Fig. 2A). At higher concentrations of IFN- $\alpha 2 \mathrm{~b}$, proliferation of Daudi/vector cells was reduced to less than $10 \%$. The specificity of the antiproliferative effects of IFN- $\alpha 2 b$ on Daudi cells was demonstrated by the lack of growth inhibition of the B lymphocyte cell line BJAB (squares), which did not respond to IFN- $\alpha 2 b$ in this assay. In contrast to controls, the proliferation of Daudi/vIRF cells (circles) was greater than $80 \%$ at IFN- $\alpha 2 \mathrm{~b}$ concentrations of 0.5 and $5.0 \mathrm{U} / \mathrm{ml}$. The resistance of Daudi/vIRF cells to the antiproliferative effects of IFN- $\alpha 2 b$ was partially overcome at concentrations of 50 and $500 \mathrm{U} / \mathrm{ml} \mathrm{IFN-}$ $\alpha 2 b$. The proliferation of Daudi/vIRF cells at 5 days after IFN treatment showed a pattern of resistance similar to that observed after 3 days of IFN treatment except that the growth of Daudi/ VIRF cells at concentrations of 50 and $500 \mathrm{U} / \mathrm{ml}$ of IFN- $\alpha 2 b$ was even greater at 5 days versus 3 days of IFN- $\alpha 2 b$ treatment (Fig. 2B). This is likely due to the degradation of IFN in the cell culture over time and subsequent growth of Daudi/vIRF cells in the pooled population of transformed cells that express high levels of vIRF. No differences in the growth rate of Daudi/vector and Daudi/vIRF cells were observed in the absence of IFN- $\alpha 2 \mathrm{~b}$ (data not shown), thus eliminating the 


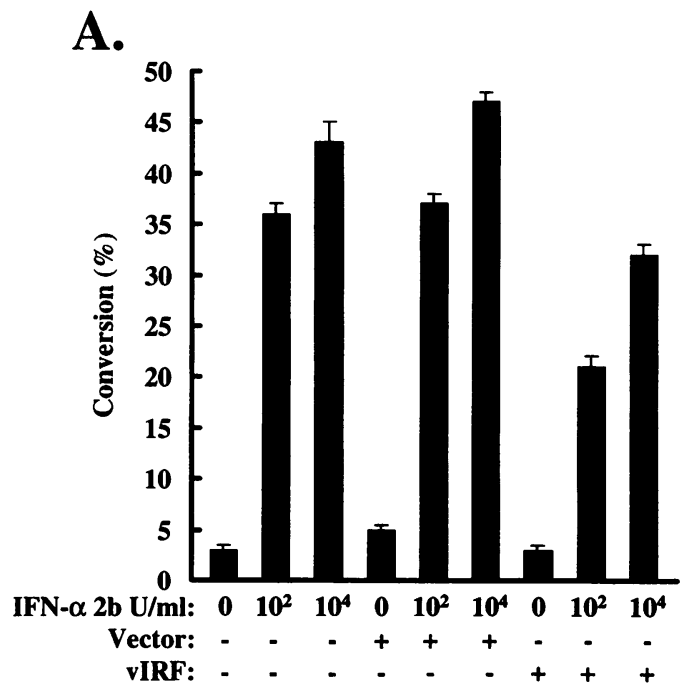

Fig. 3. vIRF specifically inhibits induction of gene expression by IFN- $\alpha$ or IRF-1. (A) HeLa cells were co-transfected with $2 \mu \mathrm{g}$ of IFN- $\beta$-CAT reporter DNA and 2,4 , or $6 \mu \mathrm{g}$ of either pMYCHIS vector DNA or pK9MH DNA. Twenty-four hours after transfection the monolayers were incubated with either 100 or 10,000 units/ml of IFN- $\alpha 2 \mathrm{~b}$ for $12 \mathrm{hr}$. Values represent the percent of chloramphenicol conversion averaged from three transfections. (B) vIRF represses IRF-1-dependent activation of the IFN- $\beta$ promoter. HeLa cells were co-transfected with $2 \mu \mathrm{g}$ of IFN- $\beta$-CAT, $2 \mu \mathrm{g}$ of pIRF-1HA, and 2,4 , or

possibility that Daudi/vIRF cells have an inherently higher rate of proliferation than the parental or Daudi/vector cells. Furthermore, the number of nonviable cells in the IFN- $\alpha$-treated cultures compared with the interferon-free controls was not significantly different, suggesting that IFN- $\alpha$ affected the rate of proliferation rather than cell viability. These data demonstrate that expression of vIRF in a human B lymphocyte cell line can overcome the growth-suppressive effect of IFN- $\alpha$.

\section{VIRF Inhibits IFN-Mediated Activation of the IFN- $\beta$ Promoter}

To examine the molecular basis of vIRF-mediated inhibition of the cellular response to IFN- $\alpha$, the capacity of vIRF to affect activation of the IFN-responsive promoter IFN- $\beta$ was analyzed. HeLa cells were transfected with the reporter construct IFN- $\beta$-CAT (48), and $24 \mathrm{hr}$ post-transfection the cells were treated with IFN- $\alpha 2 b$. Twelve hours after IFN- $\alpha 2 b$ treatment, the cells were harvested and CAT activity was quanti-
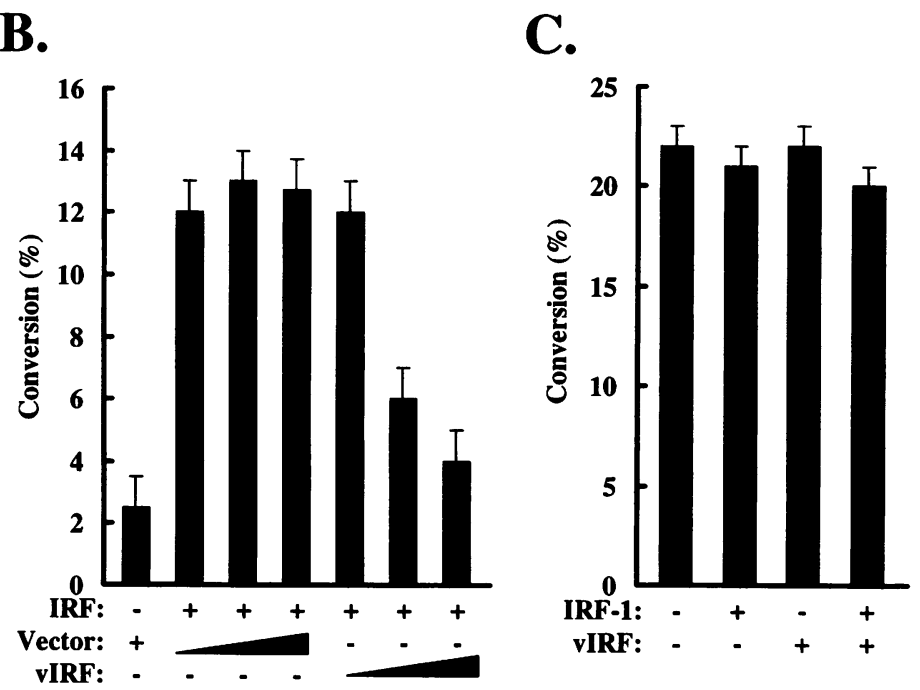

$6 \mu \mathrm{g}$ of either pMYCHIS vector or pK9MH vIRF DNAs (black triangles). Values represent the percent of acetylated chloramphenicol in the CAT assay. The data are the average CAT activity from three independent transfections. (C) vIRF does not inhibit gene expression from promoters without IRF-1 binding sites. HeLa cells were co-transfected with $2 \mu \mathrm{g}$ of RSV-CAT and $4 \mu \mathrm{g}$ of either IRF-1HA or VIRF expression vector. Transfections were conducted in triplicate, and the data are presented as the average percent conversion of chloramphenicol in each of the three CAT assays.

tated. Cells transfected with IFN- $\beta$-CAT and incubated with $100 \mathrm{U} / \mathrm{ml}$ of IFN- $\alpha 2 \mathrm{~b}$ resulted in $\sim 10$-fold activation of the IFN- $\beta$ promoter (Fig. 3A). In contrast, vIRF-transfected cells showed substantially diminished IFN- $\beta$-CAT activity in response to IFN- $\alpha 2 \mathrm{~b}$ treatment. Inhibition of IFN- $\beta$ promoter by vIRF expression was observed even when $10,000 \mathrm{U} / \mathrm{ml}$ IFN- $\alpha 2 \mathrm{~b}$ was employed. A reduction in IFN- $\beta$ promoter activity was not observed when vector plasmid DNA was substituted for vIRF plasmid, demonstrating that inhibition was not due to promoter competition from input DNAs.

\section{Inhibition of IRF-1-Dependent Transactivation by $V I R F$}

The cis-acting sequences within the IFN- $\beta$ promoter/enhancer region that are responsible for activation by IFN have been well defined (reviewed in ref. 36). The positive regulatory domain I (PRDI) contains four tandemly repeated IRF-1 binding sites that are responsible for IRF-1 binding and transactivation in response to viral 
infection or IFN- $\alpha$ signaling $(37,49)$. To determine whether vIRF inhibits IFN- $\alpha$ signaling by affecting the function of IRF-1, HeLa cells were co-transfected with IFN- $\beta$-CAT reporter, the pK9MH vIRF expression vector, or an IRF-1 expression vector plasmid, pIRF-1. That IRF-1 transactivates the IFN $-\beta$ promoter was shown by co-transfection of the IFN- $\beta$-CAT reporter with pIRF-1HA, resulting in a 5 - to 7 -fold increase in CAT activity (Fig. 3B). When increasing amounts of vIRF expression vector plasmid were co-transfected with pIRF-1 expression plasmid, the levels of CAT activity were reduced in a dose-dependent fashion. When vector DNA was substituted for the vIRF expression vector, no inhibition of pIRF-1HA transactivation was observed.

One possibility for the inhibition of IRF-1mediated activation was that expression of vIRF induced a general inhibition of transcription. To eliminate this possibility, the effects of vIRF were analyzed using as an IRF-1-independent reporter, RSV-CAT, in which the Rous sarcoma virus (RSV) promoter directs expression of the CAT gene. RSV-CAT was unresponsive to IRF-1 as shown by co-transfection of HeLa cells with pRSV-CAT and pIRF-1 DNAs (Fig. 3C). The vIRF expression vector, alone or in combination with pIRF-1, did not reduce CAT activity, thus demonstrating that vIRF does not inhibit gene expression nonspecifically.

\section{vIRF Does not Affect IRF-1 DNA Binding Activity}

Electrophoretic mobility shift assays were conducted to determine whether vIRF could inhibit the binding of IRF-1 to the ICS. As expected, IRF-1 translated in vitro binds to the ICS, as seen by the shift of radiolabeled probe to a slower migrating form (Fig. 4; lanes 1 versus 2). IRF-1 binding activity was specific for pIRF-1HA template in vitro transcription and translation reactions since reactions programmed with vector DNA did not possess ICS binding activity (Fig. 4, lane 1). The specificity of IRF-1 binding was demonstrated by competition of IRF-1 binding with cold ICS oligonucleotide (Fig. 4, lane 4) and by the lack of competition of IRF-1 binding with cold mutant ICS oligonucleotide (Fig. 4, lane 3). Unlike IRF-1, no binding activity was detected for vIRF (Fig. 4, lane 5). Furthermore, IRF-1 binding activity was not affected by the presence of VIRF (Fig. 4, compare lanes 2 and 6). These data suggest that inhibition of IRF-1 transactivation function by vIRF involves protein-protein

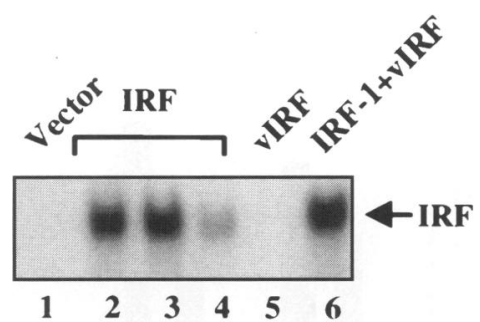

Fig. 4. vIRF does not possess detectable DNA binding activity and does not inhibit IRF-1 binding to DNA. Aliquots of in vitro transcription and translation reactions that utilized either vector, pK9MH vIRF or pIRF-1HA template plasmid DNAs, were added to binding reactions containing radiolabeled ICS oligonucleotide. IRF-1 bound to the ICS (lane 2), and this binding activity is greatly reduced in the presence of a 5-fold excess of cold competitor oligonucleotide (lane 4). IRF-1 binding was unaffected by addition of a 5-fold excess of mutant oligonucleotide (lane 3). Reactions programmed with vector DNA did not result in a mobility shift (lane 1). Incubation of VIRF with the probe does not result in a mobility shift (lane 5) nor does coincubation of vIRF and IRF- 1 alter IRF-1 binding to the probe (lane 6).

interactions that do not result in competition for binding to DNA target sites.

\section{Discussion}

In response to stimulation of cells with IFN- $\alpha$ or to virus infection, the cellular transcriptional transactivator IRF-1 increases expression of a panoply of IFN-responsive genes. One consequence of IRF-1-mediated gene activation is the inhibition of cell proliferation, as ectopic overexpression of IRF-1 suppresses cell growth in several cell types $(50,51)$. In this report, KSHV VIRF was demonstrated to confer resistance to growth suppression by IFN- $\alpha$ in a human B lymphocyte cell line, to inhibit activation of an IFN-responsive promoter by stimulation of cells with IFN- $\alpha$, and to repress IRF-1-dependent transactivation. IRF-l transactivation is subject to regulation by other IRF family members that share homology within an amino-terminal DNA binding domain (35). IRF-2 and ICSAT repress IRF- 1 transactivation function by competition for ICS binding sites (37-39,52). Repression of IRF-1 function by ICSBP may involve protein-protein interactions between ICSBP and IRF-1 $(53,54)$ or competition with IRF-1 for ICS binding sites $(55,56)$. The molecular mechanism by which vIRF inhibits IRF-1 function is apparently novel as these stud- 
ies did not reveal interactions between vIRF and IRF-1 (data not shown) nor binding activity of vIRF to the ICS (Fig. 4). A recent study has also reported that vIRF inhibits both IFN responses and IRF-1-mediated transactivation (40), as described here. In concordance with the studies described in this report, vIRF did not inhibit IRF-1 binding to DNA nor did vIRF bind directly to IRF-1 (40). These data suggest that vIRF may employ a mechanism of repression that differs from that of cellular IRF family members.

The recent findings that vIRF is a transforming oncoprotein when assayed in murine fibroblasts suggests that vIRF may play a role in the induction of tumorigenesis in vivo. vIRF gene expression has been detected in cell lines derived from BCBL but not KS tumor tissues, raising the possibility that vIRF may contribute to the induction of B lymphomas associated with KSHV. The EBV EBNA-2 polypeptide, when expressed in Daudi cells or in EBV-negative Burkitt's lymphoma cell lines, imparts resistance to IFN- $\alpha$ mediated growth inhibition (46), a function similar to that described for vIRF. EBNA-2 may affect IFN signaling by decreasing levels of ISGF3 (47), a complex of three polypeptides (STAT-1, STAT-2, and p48) that activates promoters containing the IFN-stimulated response element. The observation that vIRF inhibited activation of the interferon-stimulated response element by IRF-1 more effectively than the interferon-stimulated response element by IFN- $\alpha$ suggests that vIRF may show relative specificity for IRF-1, rather than p48-STAT-1-STAT-2, activation. Thus, EBV and KSHV, two B lymphotropic herpesviruses, have encoded viral proteins whose function is to suppress growth inhibition by IFN- $\alpha$, albeit by targeting different components of IFN-signaling pathways. Interestingly, EBNA-2 in conjunction with other EBV proteins is required for the immortalization of primary $B$ lymphocytes (57). The association of KSHV with two B lymphocyte neoplasms, body cavity-based lymphoma and multicentric Castleman's disease $(20-23)$, suggests that KSHV may cause dysregulation of $\mathrm{B}$ lymphocyte proliferation in part through this mechanism.

The expression of a viral protein capable of inhibiting IRF-1-mediated activation of IFN-responsive genes may provide KSHV with an advantage in countering cellular responses to viral infection. Gene targeting of IRF-1 revealed that IRF-1-deficient mice are less resistant to infection by encephalomyocarditis virus infection (58). In addition, IRF- 1 is implicated in the acti- vation of immune response genes such as class I histocompatibility antigens and in the activation of regulators of cell growth such as p21, doublestranded RNA-dependent protein kinase (PKR), and $2^{\prime} 5^{\prime}$-oligoadenylate synthetase (35). IRF-1 plays a role in programmed cell death since normal embryonic fibroblasts, but not IRF- ${ }^{-1-}$ embryonic fibroblasts, undergo apoptosis in response to expression of oncogenic ras combined with a block to cell proliferation (59). Also, IRF-1 is required for DNA damage-induced apoptosis of mature $T$ lymphocytes (60). Thus, IRF-1 plays a role in the regulation of cell proliferation, the immune response, and programmed cell death, all of which are potential responses to viral infection.

IRF-1 has tumor-suppressor functions, since activated $\mathrm{c}-\mathrm{H}$-ras is sufficient to transform embryonic fibroblasts from IRF-1-deficient mice but is not sufficient to transform normal embryonic fibroblasts. Deletions within the IRF-1 locus have been identified in leukemias and myelodysplastic syndrome, suggesting that loss of IRF-1 anti-oncogenic function may be associated with tumorigenicity in vivo (61). The findings that VIRF inhibits the transactivation function of IRF-1 $(40,41)$, a known tumor suppressor protein, that vIRF transforms NIH3T3 cells (41), and that vIRF confers resistance to IFN- $\alpha$-mediated suppression of cell growth in a cell type relevant to KSHV infection suggest that expression of vIRF in KSHV-infected B lymphocytes may affect numerous cellular responses to viral infection and/or cytokine stimulation, including cellular transformation.

\section{Acknowledgments}

We thank Ms. Donna Gschwend and Ms. Nancy Barrett for manuscript preparation. This work was supported in part by a grant from the National Institutes of Health (CA46592).

\section{References}

1. Chang Y, Cesarman E, Pessin MS, et al. (1994) Identification of herpesvirus-like DNA sequences in AIDS-associated Kaposi's sarcoma. Science 266: 1865-1869.

2. Ambroziak JA, Blackburn DJ, Herndier BG, et al. (1995) Herpes-like sequences in HIV-infected and uninfected Kaposi's sarcoma patients. Science 268: 582-583.

3. Boshoff C, Whitby D, Hatziioannou T, et al. (1995) 
Kaposi's-sarcoma-associated herpesvirus in HIVnegative Kaposi's sarcoma. Lancet 345: 1043-1044.

4. Dupin N, Grandadam M, Calvez V, et al. (1995) Herpesvirus-like DNA sequences in patients with Mediterranean Kaposi's sarcoma. Lancet 345: 761762.

5. Moore PS, Chang Y. (1995) Detection of herpeslike virus DNA sequences in Kaposi's sarcoma in patients with and without HIV infection. $N$. Engl. J. Med. 332: 1181-1185.

6. Schalling M, Ekman M, Kaaya EE, Linde A, Biberfeld P. (1995) A role for a new herpes virus (KSHV) in different forms of Kaposi's sarcoma. Nat. Med. 1: 707-708.

7. Gao SJ, Kingsley L, Hoover DR, et al. (1996) Seroconversion to antibodies against Kaposi's sarcoma-associated herpesvirus-related latent nuclear antigens before the development of Kaposi's sarcoma. N. Engl. J. Med. 335: 233-241.

8. Moore PS, Kingsley LA, Holmberg SD, et al. (1996) Kaposi's sarcoma-associated herpesvirus infection prior to onset of Kaposi's sarcoma. AIDS 10: $175-180$.

9. Whitby D, Howard MR, Tenant-Flowers M, et al. (1995) Detection of Kaposi sarcoma associated herpesvirus in peripheral blood of HIV-infected individuals and progression to Kaposi's sarcoma. Lancet 346: 799-802.

10. Kedes DH, Operskalski E, Busch M, Kohn R, Flood J, Ganem D. (1996) The seroepidemiology of human herpesvirus 8 (Kaposi's sarcoma-associated herpesvirus): distribution of infection in KS risk groups and evidence for sexual transmission. Nat. Med. 2: 918-924.

11. Lennette ET, Blackboum DJ, Levy JA. (1996) Antibodies to human herpesvirus type 8 in the general population and in Kaposi's sarcoma patients. Lancet 348: 858-861.

12. Simpson GR, Schulz TF, Whitby D, et al. (1996) Prevalence of Kaposi's sarcoma associated herpesvirus infection measured by antibodies to recombinant capsid protein and latent immunofluorescence antigen. Lancet 348: 1133-1138.

13. Boshoff C, Schulz TF, Kennedy MM, et al. (1995) Kaposi's sarcoma-associated herpesvirus infects endothelial and spindle cells. Nat. Med. 1: 12741278.

14. Decker LL, Shankar P, Khan G, et al. (1996) The Kaposi sarcoma-associated herpesvirus (KSHV) is present as an intact latent genome in $\mathrm{KS}$ tissue but replicates in the peripheral blood mononuclear cells of KS patients. J. Exp. Med. 184: 283-288.

15. Zhong W, Wang H, Herndier B, Ganem D. (1996) Restricted expression of Kaposi sarcoma-associated herpesvirus (human herpesvirus 8) genes in Kaposi sarcoma. Proc. Natl. Acad. Sci. U.S.A. 93: 6641-6646.
16. Orenstein JM, Alkan S, Blauvelt A, et al. (1996) Visualization of human herpesvirus type 8 in $\mathrm{Ka}$ posi's sarcoma by light and transmission electron microscopy. AIDS 11: F35-F45.

17. Foreman KE, Friborg J Jr, Kong W, et al. (1997) Propagation of a human herpesvirus from AIDSassociated Kaposi's sarcoma. N. Engl. J. Med. 336: 163-171.

18. Moore PS, Boshoff C, Weiss RA, Chang Y. (1996) Molecular mimicry of human cytokine and cytokine response pathway genes by KSHV. Science 274: 1739-1744.

19. Russo JJ, Bohenzky RA, Chien MC, et al. (1996) Nucleotide sequence of the Kaposi sarcoma-associated herpesvirus (HHV8). Proc. Natl. Acad. Sci. U.S.A. 93: 14862-14867.

20. Arvanitakis L, Mesri EA, Nador RG, et al. (1996) Establishment and characterization of a primary effusion (body cavity-based) lymphoma cell line (BC-3) harboring Kaposi's sarcoma-associated herpesvirus (KSHV/HHV-8) in the absence of Epstein-Barr virus. Blood 88: 2648-2654.

21. Cesarman E, Chang Y, Moore PS, Said JW, Knowles DM. (1995) Kaposi's sarcoma-associated herpesvirus-like DNA sequences in AIDS-related body-cavity-based lymphomas. N. Engl. J. Med. 332: 1186-1191.

22. Renne R, Zhong W, Herndier B, et al. (1996) Lytic growth of Kaposi's sarcoma-associated herpesvirus (human herpesvirus 8 ) in culture. Nat. Med. 2: 342-347.

23. Soulier J, Grollet L, Oksenhendler E, Cacoub P, Cazals-Hatem D, Babinet P. (1995) Kaposi's sarcoma-associated herpesvirus-like DNA sequences in multicentric Castleman's disease. Blood 86: 1276-1280.

24. Moore PS, Gao SJ, Dominguez G, et al. (1996) Primary characterization of a herpesvirus agent associated with Kaposi's sarcoma. J. Virol. 70: $549-558$.

25. Roizman, B, Sears, AE. (1995) Herpes Simplex Viruses and Their Replication. In: Fields BN, Knipe DM, Howley PM (eds). Fields Virology, Third Edition, Lippincott-Raven, Philadelphia, pp. 2231-2296.

26. Rickinson, AB, Kieff, E. (1995) Epstein-Barr Virus. In: Fields BN, Knipe DM, Howley PM (eds). Fields Virology, Third Edition, Lippincott-Raven, Philadelphia, pp. 2397-2446.

27. Jung JU, Desrosiers RC. (1995) Association of the viral oncoprotein STP-C488 with cellular ras. $\mathrm{Mol}$. Cell. Biol. 15: 6506-6512.

28. Meinl E, Hohlfeld R, Wekerle H, Fleckenstein B. (1995) Immortalization of human $T$ cells by herpesvirus saimiri. Immunol. Today 16: 55-58.

29. Godden-Kent D, Talbot SJ, Boshoff C, et al. (1997) The cyclin encoded by Kaposi's sarcoma-associated herpesvirus stimulates cdk6 to phosphorylate the retinoblastoma protein and histone $\mathrm{Hl}$. J. Virol. 71: 4193-4198. 
30. Li M, Lee H, Yoon D, et al. (1997) Kaposi's sarcoma-associated herpesvirus encodes a functional cyclin. J. Virol. 71: 1984-1991.

31. Sarid R, Sato T, Bohenzky RA, Russo JJ, Chang Y. (1997) Kaposi's sarcoma-associated herpesvirus encodes a functional Bcl-2 homologue. Nat. Med. 3: 293-298.

32. Bertin J, Armstrong RC, Ottilie S, et al. (1997) Death effector domain-containing herpesvirus and poxvirus proteins inhibit both Fas- and TNFR l-induced apoptosis. Proc. Natl. Acad. Sci. U.S.A. 94: 1172-1176.

33. Thome M, Schneider P, Hofmann K, et al. (1997) Viral FLICE-inhibitory proteins (FLIPs) prevent apoptosis induced by death receptors. Nature 386: 517-521

34. Rettig MB, Ma HJ, Vescio RA, et al. (1997) Kaposi's sarcoma-associated herpesvirus infection of bone marrow dendritic cells from multiple myeloma patients. Science 276: 1851-1854.

35. Yamamoto H, Lamphier MS, Fujita T, Taniguchi T, Harada H. (1994) The oncogenic transcription factor IRF-2 possesses a transcriptional repression and a latent activation domain. Oncogene 9: 14231428.

36. Taniguchi $T$, Harada H, Lamphier M. (1995) Regulation of the interferon system and cell growth by IRF transcription factors. J. Cancer Res. Clin. Oncol. 121: 516-520.

37. Tanaka N, Taniguchi T. (1992) Cytokine gene regulation: regulatory cis-elements and DNA binding factors involved in the interferon system. $A d v$. Immunol. 52: 263-281.

38. Harada H, Fujita T, Miyamoto M, et al. (1989) Structurally similar but functionally distinct factors, IRF-1 and IRF-2, bind to the same regulatory elements of IFN and IFN-inducible genes. Cell 58: 729-739.

39. Harada H, Willison $K$, Sakakibara J, Miyamoto $M$, Fujita T, Taniguchi T. (1990) Absence of the type I IFN system in EC cells: transcriptional activator (IRF-1) and repressor (IRF-2) genes are developmentally regulated. Cell 63: 303-312.

40. Zimring JC, Goodbourn S, Offermann MK. (1998) Human herpesvirus 8 encodes an interferon regulatory factor (IRF) homolog that represses IRF1-mediated transcription. J. Virol. 72: 701-707.

41. Gao S-J, Boshoff C, Jayachandra S, Weiss RA, Chang Y, Moore PS. (1997) KSHV ORF K9 (vIRF) is an oncogene which inhibits the interferon signaling pathway. Oncogene 15: 1979-1985.

42. Kozak M. (1986) Point mutations define a sequence flanking the AUG initiator codon that modulates translation by eukaryotic ribosomes. Cell 44: 283-292.

43. Kingston RE. (1989) CaPO4 Transfection. In: Ausebel FM, Brent R, Kingston RE, Moore DD, Seidman JG, Smith JA, Struhl K (eds). Current Protocols in Molecular Biology, John Wiley \& Sons, NY, pp. 9.1.1-9.1.4.
44. Lin R, Mustafa A, Nguyen H, Gewert D, Hiscott J. (1994) Mutational analysis of interferon (IFN) regulatory factors 1 and 2 : effects on the induction of IFN-beta gene expression. J. Biol. Chem. 269: 17542-17549.

45. Maniatis T, Jeffrey A, van de Sande H. (1975) Chain length determination of small double- and single-stranded DNA molecules by polyacrylamide gel electrophoresis. Biochemistry 14: 37873794.

46. Aman P, von Gabain A. (1990) An Epstein-Barr virus immortalization associated gene segment interferes specifically with the IFN-induced antiproliferative response in human B-lymphoid cell lines. $E M B O$ J. 9: 147-152.

47. Kanda K, Decker T, Aman P, Wahlstrom M, von Gabain A, Kallin B. (1981) The EBNA2-related resistance towards alpha interferon (IFN-alpha) in Burkitt's lymphoma cells effects induction of IFNinduced genes but not the activation of transcription factor ISGF-3. Mol. Cell. Biol. 12: 4930-4936.

48. Thanos D, Maniatis T. (1995) Identification of the rel family members required for virus induction of the human beta interferon gene. Mol. Cell. Biol. 15: 152-164.

49. Fujita T, Sakakibara J, Sudo Y, Miyamoto M, Kimura Y, Taniguchi T. (1988) Evidence for a nuclear factor(s), IRF-1, mediating induction and silencing properties to human IFN-beta gene regualtory elements. EMBO J. 7: 3397-3405.

50. Harada H, Takahashi E, Itho S, Harada K, Hori TA, Taniguchi T. (1994) Structure and regulation of the human interferon regulatory factor 1 (IRF-1) and IRF-2 genes: implications for a gene network in the interferon system. Mol. Cell. Biol. 14: 1500-1509.

51. Kirchhoff S, Schaper F, Hauser H. (1993) Interferon regulatory factor 1 (IRF-1) mediates cell growth inhibition by transactivation of downstream target genes. Nucl. Acids Res. 21: 2881-2889.

52. Yamagata T, Nishida J, Tanaka S, et al. (1996) A novel interferon regulatory factor family transcription factor, ICSAT/Pip/LSIRF, that negatively regulates the activity of interferon-regulated genes. Mol. Cell. Biol. 16: 1283-1294.

53. Bovolenta C, Driggers PH, Marks MS, et al. (1994) Molecular interactions between interferon consensus sequence binding protein and members of the interferon regulatory factor family. Proc. Natl. Acad. Sci. U.S.A. 91: 5046-5050.

54. Sharf R, Azriel A, Lejbkowicz F, Winograd SS, Ehrlich R, Levi BZ. (1995) Functional domain analysis of interferon consensus sequence binding protein (ICSBP) and its association with interferon regulatory factors. J. Biol. Chem. 270: 13063-13069.

55. Nelson N, Marks MS, Driggers PH, Ozato K. (1993) Interferon consensus sequence-binding protein, a member of the interferon regulatory factor family, suppresses interferon-induced gene transcription. Mol. Cell. Biol. 13: 588-599.

56. Weisz A, Marx P, Sharf R, et al. (1992) Human 
interferon consensus sequence binding protein is a negative regulator of enhancer elements common to interferon-inducible genes. J. Biol. Chem. 267: 25589-25596.

57. Kieff, E. (1995) Epstein-Barr Virus and Its Replication. In: Fields BN, Knipe DM, Howley PM (eds). Fields Virology, Third Edition, LippincottRaven, Philadelphia, pp. 2343-2396.

58. Kimura T, Nakayama K, Penninger J, et al. (1994) Involvement of the IRF-1 transcription factor in antiviral responses to interferons. Science 264: 1921-1924.
59. Tanaka N, Ishihara M, Kitagawa M, et al. (1994) Cellular commitment to oncogene-induced transformation or apoptosis is dependent on the transcription factor IRF-1. Cell 77: 829-839.

60. Tamura T, Ishihara M, Lamphier MS, et al. (1995) An IRF-1-dependent pathway of DNA damageinduced apoptosis in mitogen-activated T lymphocytes. Nature 376: 596-599.

61. Willman CL, Sever CE, Pallavicini MG, et al. (1993) Deletion of IRF-1, mapping to chromosome 5q31.1, in human leukemia and preleukemic myelodysplasia. Science 259: 968-971. 